\title{
Redes sociais, acesso e regulação dos serviços de saúde em um município de pequeno porte do Rio de Janeiro, Brasil
}

\author{
Social networks, access and regulation of health services \\ in a small city in the state of Rio de Janeiro, Brazil
}

Juliete dos Santos Amaral Fonseca ${ }^{1}$

Helena Maria Scherlowski Leal David ${ }^{1}$

Tarciso Feijó da Silva ${ }^{1}$

Tatiana Cabral da Silva Ramos ${ }^{1}$

Ana Carolina Lopes Neves ${ }^{1}$

Rogério Bittencourt de Miranda ${ }^{1}$

${ }^{1}$ Programa de Pós-

Graduação em Enfermagem,

Universidade do Estado do

Rio de Janeiro. Boulevard

28 de Setembro $157 / 7^{\circ}$, Vila Isabel. 20551-030 Rio de

Janeiro RJ Brasil.

julietesa@outlook.com

\begin{abstract}
The scope of this study was to establish the social relationships of primary health care workers for the regulation of health care from the social network perspective. It is a descriptive study using a quantitative approach in four basic care units, the control and evaluation sector, out-ofhome treatment and basic care coordination. The data collection was performed via semi-structured interviews between December 2015 and January 2016 and the data were analyzed by Social Network Analysis with the support of the Ucinet and Netdraw softwares that allowed the analysis of the metrics and the creation of the sociogram. In the reticular dynamics, the actors with the greatest centrality of degree and intermediation were control and evaluation, coordinator of the basic care and coordinator of Out-of-Home Treatment. The nurse and the Community Health Agent emerged as central in relation to intermediation, which explains their relevance as articulators in the network. The way in which the network is articulated and the dynamics of social relations between the teams are reflected in the quality of the service and in the continuity of care. Replacing the community agents in the family health teams marks a setback because it will impact the social relations of these actors with the population.
\end{abstract}

Key words Social networking, Health services accessibility, Primary health care, Nursing
Resumo O objetivo deste estudo foi conhecer as relações sociais dos trabalhadores em saúde da atenção básica para a regulação da assistência à saúde na perspectiva da rede social. Estudo descritivo com abordagem quantitativa em quatro unidades de atenção básica, setor controle e avaliação, tratamento fora de domicílio e coordenadoria de atenção básica. A coleta de dados foi realizada pela entrevista semiestruturada entre dezembro de 2015 e janeiro de 2016, e os dados foram analisados pela Análise de Redes Sociais com apoio dos softwares Ucinet e Netdraw, que permitiram a análise das métricas e a criação do sociograma. Na dinâmica reticular os atores com maior centralidade de grau e de intermediação foram controle e avaliação, coordenador da atenção básica e coordenador do Tratamento Fora de Domicílio. O enfermeiro e o Agente Comunitário de Saúde emergiram como central em relação à intermediação, o que explicita a sua relevância como articuladores na rede. A forma com que a rede se articula e a dinâmica das relações sociais entre as equipes refletem na qualidade do serviço e na continuidade do cuidado. A substituição dos agentes comunitários nas equipes de saúde da família marca um retrocesso pois influencia nas relações sociais destes atores com a população.

Palavras-chave Redes sociais, Acesso aos serviços de saúde, Atenção primária à saúde, Enfermagem 


\section{Introdução}

O termo redes sugere dinamicidade e movimento, pois se transforma a cada instante e é dependente das interações sociais ${ }^{1}$. É um processo social amplo que forma uma estrutura social que, dependendo dos atores envolvidos, tem objetivos, valores e metas distintas ${ }^{2}$.

As redes sociais podem ser presenciais ou virtuais. Estas convergem no que tange às trocas informacionais, e diferem no modo como as trocas acontecem. Diferentemente das redes virtuais, nas presenciais não ocorre mediação de páginas na internet, pois são as interações interpessoais as responsáveis pela circulação das informações ${ }^{3}$. As redes permitem compreender a sociedade relacional assim como as trocas e compartilhamentos mediados por esta 4 .

O estudo sobre redes e suas implicações para a saúde ampliou-se a partir da compreensão da saúde na sua dimensão social ${ }^{5,6}$. Os profissionais de saúde integram diversas redes, incluindo as constituídas em seus espaços laborais, que podem ter um papel de relevância na regulação do acesso aos serviços de saúde e no atendimento às necessidades em saúde da população.

O sistema de regulação em saúde visa a garantia e o direcionamento formal do acesso aos serviços públicos na saúde ${ }^{7}$. A regulação assistencial ou regulação do acesso é voltada para o conjunto de atividades e ações em saúde tendo como objetos a organização, o controle, o gerenciamento e a priorização do acesso e dos fluxos assistenciais no âmbito do Sistema Único de Saúde (SUS) ${ }^{8}$.

A regulação tem sido considerada um desafio. Assim, a inoperância ou insuficiência do Estado (esfera federal) é apontada como a principal dificuldade para o acesso aos serviços de saúde nos níveis secundário e terciário9. Como uma forma de enfrentamento destes obstáculos, destaca-se a relevância das interações entre os profissionais, visando garantir o acesso dos usuários aos serviços de saúde?.

Nesse sentido, quando as redes são mobilizadas para garantir o acesso, os profissionais estão não apenas buscando dar conta de aspectos operacionais do seu trabalho, como também buscando efetivar princípios SUS, como acesso universal e equidade - que se relacionam com a prestação de uma assistência justa, única e singular, de acordo com a necessidade de saúde de cada indivíduo. Assim, o recorte teórico e metodológico para conhecer e analisar as redes sociais como espaço de trocas materiais, informacionais e/ou simbólicas, formais e informais, permite apreender elementos capazes de apontar para fragilidades ou potencialidades para a operacionalização dos serviços de saúde.

O objetivo deste artigo é analisar as relações sociais em rede de trabalhadores de saúde da atenção básica e seu papel nos processos de regulação da assistência, com base na perspectiva metodológica da Análise de Redes Sociais.

O estudo integra um projeto de pesquisa intitulado Redes Sociais de Enfermeiros na Atenção Básica - estudo comparativo entre o Rio de Janeiro e Ceará, financiado pelo CNPq por meio do Edital Universal 2013, e compõe a Linhas de Pesquisa "Trabalho, Educação e formação em Saúde e Enfermagem" do Programa de Pós-Graduação em Enfermagem da Universidade do Estado do Rio de Janeiro (UERJ), por meio do Grupo de Pesquisa "Configurações do Trabalho, Saúde dos Trabalhadores e Enfermagem” do CNPq.

\section{Metodologia}

A metodologia aplicada de Análise de Redes Sociais (ARS) visa mapear as interações entre os atores sociais. Para isso, através de um software, calcula-se métricas e gera-se gráficos que auxiliam a entender os papéis, fluxos de informação e trocas dentro de uma determinada rede ${ }^{10}$. Trata-se de um estudo descritivo com abordagem quantitativa, de ARS, que possibilita uma visão estrutural e relacional da rede.

O estudo foi realizado em 4 unidades de atenção básica, e 3 setores administrativos da Secretaria Municipal de Saúde (SMS)de um município de pequeno porte da Região Médio Paraíba/RJ, durante os meses de dezembro de 2015 e janeiro de 2016. O Instituto Brasileiro de Geografia e Estatística (IBGE), no Censo de 2010, convencionou que os Municípios podem ser: de pequeno porte 1 - até 20 mil habitantes, pequeno porte 2 - 20.001 até 50.000 habitantes ${ }^{11}$.

A região do Médio Paraíba/RJ conta com 12 municípios: Barra do Piraí, Barra Mansa, Itatiaia, Pinheiral, Piraí, Porto Real, Quatis, Resende, Rio Claro, Rio das Flores, Valença e Volta Redonda. O município estudado, tem uma população inferior a 20.000 habitantes e uma extensão territorial de $50.779 \mathrm{Km}^{2}$, sendo, portanto, um município de pequeno porte. O mesmo município possui um sistema de transporte público que circula por toda cidade gratuitamente todos os dias, o que facilita o acesso geográfico das pessoas às unidades de saúde. 
Além de 7 unidades básicas de saúde, o município detém centro de diagnóstico, centro de especialidades odontológicas, central de fisioterapia e reabilitação, ambulatório de saúde mental e Centro de Atenção Psicossocial (CAPS), com uma cobertura populacional de Estratégia Saúde da Família (ESF) de 100\%.

O município revela um perfil sociodemográfico e de saúde superior ao da maioria dos municípios pequenos e médios do país, com bons indicadores de cobertura de saneamento básico e de serviços de saúde.

O cálculo do coeficiente de mortalidade por 1000 habitantes permite observar taxas de 3,32, em 2011, e 3,24, em 2015. Esse dado, associado ao crescimento populacional, que em 2011 era de 16.253 e em de 2015 de 17.272 , possibilita compreender que não houve mudança significativa no número de óbitos no decorrer dos anos. Pressupõe-se que as taxas com valores baixos podem estar relacionadas à organização dos serviços de saúde, às boas condições socioeconômicas do município e à cobertura de $100 \%$ de $\mathrm{ESF}^{12}$. Optou-se por abordar o número de óbitos no município estudado em um dado período pois este é um indicador que, além de fornecer dados brutos do quantitativo de óbitos relacionados às causas e idade, permite conhecer e compreender a situação de saúde e a organização e implementação das políticas de saúde no município.

No que diz respeito às causas dos óbitos, percebe-se que as mesmas refletem a realidade de outros municípios do Rio de Janeiro, estando associadas às doenças não transmissíveis. São elas, doenças do sistema circulatório, seguidas das neoplasias, acometendo pessoas a partir dos 50 anos de idade, sendo mais freqüente entre 60 a 79 $\operatorname{anos}^{12}$. Nessa perspectiva, considera-se o envelhecimento populacional, a transição demográfica e a transição epidemiológica para compreender esses dados. E ainda as vulnerabilidades da pessoa idosa e suas suscetibilidades às doenças.

O aumento da renda per capita se deu após os investimentos no setor da indústria automobilística na Região do Médio Paraíba, durante a primeira metade da década de 2010, o que levou a um aumento expressivo da renda média e da empregabilidade. Estes investimentos acompanharam a expansão da rede de serviços de saúde, incluindo os serviços especializados.

Participaram da pesquisa, 4 enfermeiros, 1 médico, 3 Agentes Comunitários de Saúde (ACS), 1 coordenador da atenção básica (também enfermeiro) e 3 administradores (1 do controle e avaliação e 2 do Tratamento Fora do Domicílio
(TFD)), totalizando 12 profissionais. Foram adotados como critérios de inclusão participantes com mais de seis meses de vínculo empregatício, maiores de 18 anos, e que atuassem em unidades de atenção básica ou em setores que estivessem relacionados com as referências e encaminhamentos para outros níveis de atenção à saúde.

A técnica para obtenção dos dados foi entrevista semiestruturada, na qual utilizou-se um roteiro com perguntas para nortear os relatos sobre os encaminhamentos para a atenção especializada, com gravação, transcrição e posterior análise.

Conforme o método de ARS, os participantes citaram até cinco pessoas relacionadas ao processo de encaminhamento dos usuários para a rede especializada e para garantia do acesso dos mesmos a esses serviços.

A organização e análise dos dados contou com o apoio do software Ucinet $\subset$ e sua extensão, Netdraw@, para produção de uma matriz quadrada e cálculo das medidas de centralidade ${ }^{13-15}$. Posteriormente, os dados foram importados para o Netdraw para gerar os sociogramas. A partir deste momento, analisa-se a posição de cada ator social na rede, sua influência, dada pelas medidas de centralidade, e as relações entre os participantes.

Esta pesquisa foi pautada nos critérios que regulamentam os aspectos ético-legais da pesquisa envolvendo seres humanos, definidos na Resolução no 466/2012, do Conselho Nacional de Saúde (CNS), e obteve aprovação do Comitê de Ética em Pesquisa da UERJ, com anuência institucional da SMS do município estudado.

\section{Resultados e discussão}

A representação gráfica da rede foi obtida a partir das perguntas sobre contatos ou profissionais estratégicos na garantia e efetivação do acesso às redes de atenção especializada (Figura 1).

Este grafo possui um total de 28 nós (atores) e 37 arestas ou ligações (setas), com uma densidade de 0,049; em termos percentuais, 4,9\%. A densidade é uma métrica que avalia a conectividade dos nós dentro de uma rede ${ }^{16}$. Uma rede ou grafo completo tem todas as arestas possíveis e densidade igual a 1, ou seja, é aquele em que, para cada nó, existe uma aresta conectando esse nó aos demais; ou seja, é um grafo em que todos os seus componentes encontram-se conectados, isto é, quanto maior o número de ligações, mais densa é a rede $\mathrm{e}^{10,17}$.

A densidade é uma medida de conectividade dentro da rede que informa a respeito de sua for- 


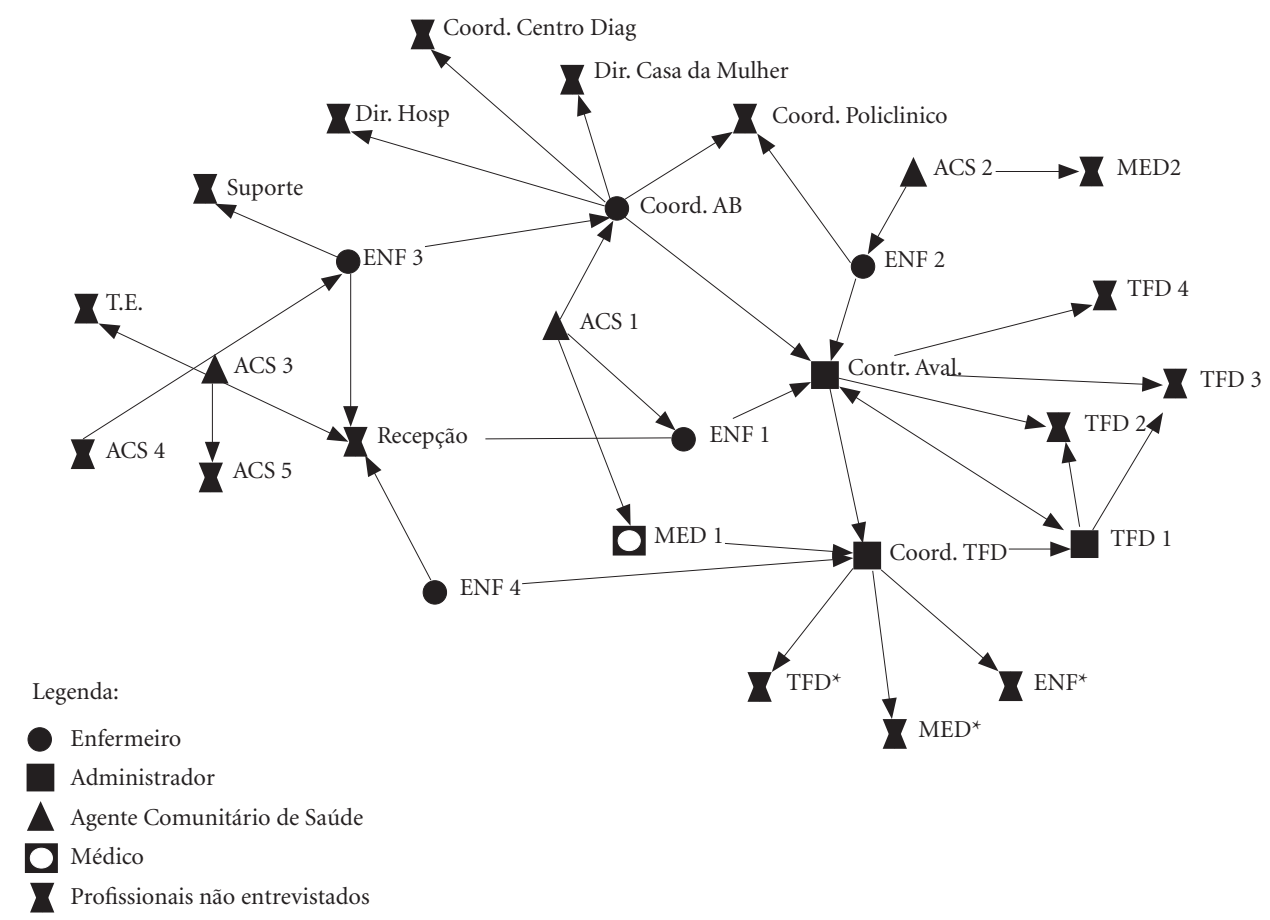

Figura 1. Atores envolvidos na Atenção Básica para o acesso à saúde segundo a categoria profissional.

Fonte: Autores, 2016.

ça como rede capaz de interligar todos os atores, as redes mais conectadas possuem muitas ligações e quanto maior essa medida, maior a coesão do grupo ${ }^{16}$.

Esta rede apresentou baixa conectividade. Isto significa que não há facilitação na comunicação direta entre todos os atores, e que pode haver a tendência de concentrar (centralizar) o fluxo informacional em atores específicos ${ }^{10,18}$. Em se tratando de uma rede socioprofissional, e num dos setores onde ocorre maior rotatividade da força de trabalho, é um resultado que não chega a surpreender, por um lado. Por outro lado, seria de se esperar que as equipes da atenção básica possuíssem fluxo de comunicação bastante estreitado entre os profissionais, dado o caráter integrador da proposta da Política Nacional de Atenção Básica (PNAB) ${ }^{19}$.

Através da representação da rede é possível observar que os enfermeiros possuem contato com responsáveis de outras unidades de saúde, tornando-se, assim, um elo importante na rede, até mesmo como mediador entre os demais profissionais. Como o município trabalha com cotas,o setor controle e avaliação aparece como importante para o acesso, pois faz a gestão dessas cotas para a rede especializada de atenção dentro do município.

Caso não haja alguma especialidade no município, o TFD realiza os contatos para fora do município para agendar exames e procedimentos. Essas marcações acontecem via Sistema Estadual de Regulação (SER) e Sistema Nacional de Regulação (SISREG). O município, na maioria dos casos, faz o transporte do usuário para os locais referenciados, o que facilita a assistência integral e longitudinal.

Outro destaque na garantia do acesso é a recepção, pois através dela ocorre o primeiro contato dos usuários com a unidade básica. Esses profissionais, em geral, realizam contato com o TFD, controle e avaliação, secretaria de saúde, coordenação da policlínica e suporte para o sistema de informática utilizado dentro do município.

Já os enfermeiros tendem a ser mediadores dentro da equipe, e isto se justifica, em parte, pelo duplo caráter do seu processo de trabalho - gerencial e assistencial ${ }^{20}$. Também o fato de possuírem cargas horárias contratuais diferentes dos médicos, e sua capacidade para a tomada de de- 
cisões dentro das ações da atenção básica acabam por colocar o enfermeiro em lugar de destaque na rede. Questão também observada em um estudo canadense com equipes de atenção primária usando a $\mathrm{ARS}^{21}$.

Em relação aos ACS, todos citaram o enfermeiro como referência para suporte, logística, conhecimento e para sanar possíveis dúvidas sobre atendimento e processo de trabalho, reforçando o papel do enfermeiro na supervisão e educação permanentes.

\section{Centralidade de grau}

A centralidade de grau é definida pelo número de contatos diretos que um ator tem com os demais elos da rede. É um canal direto de informação, cuja medida é dividida em grau de entrada e saída, dependendo da direção dos fluxos ${ }^{17}$. O grau de entrada (indegree) é o número de links que chegam a um nó. Quando um ator tem muitas ligações direcionadas a ele, isto indica que é um ator importante ou com prestígio na rede. Já o grau de saída (outdegree) é o número de links que saem de um nó, ou seja, é o ator que procura outros $^{22}$. Os valores de centralidade de grau consistem no somatório dos graus de entrada e saída.

Os atores com maior centralidade de grau são os que, na Figura 2, aparecem com seus símbolos em tamanho superior aos demais nós da rede. São eles: Controle e avaliação, Coordenador da atenção básica e Coordenador do TFD, os gestores organizacionais. Do ponto de vista da política de regulação, este fato era esperado, evidenciando que os setores possuíam capacidade para efetivar suas atribuições no processo de regulação ${ }^{23}$.

No Quadro 1, seguem as medidas de centralidade de grau dos atores desta rede, separando os graus de entrada e saída.

Considerando os graus de entrada, o controle e avaliação, coordenação TFD e recepção possuem maiores centralidades.

A portaria no 423/2002, do Ministério da Saúde $^{23}$ (MS) aprova as atribuições básicas inerentes a cada nível do governo no controle, avaliação e regulação da assistência à saúde no SUS. Compete ao MS oferecer condições para os estados e municípios organizarem seus sistemas de saúde para garantir o acesso às ações e serviços que atendam com qualidade e resolubilidade os

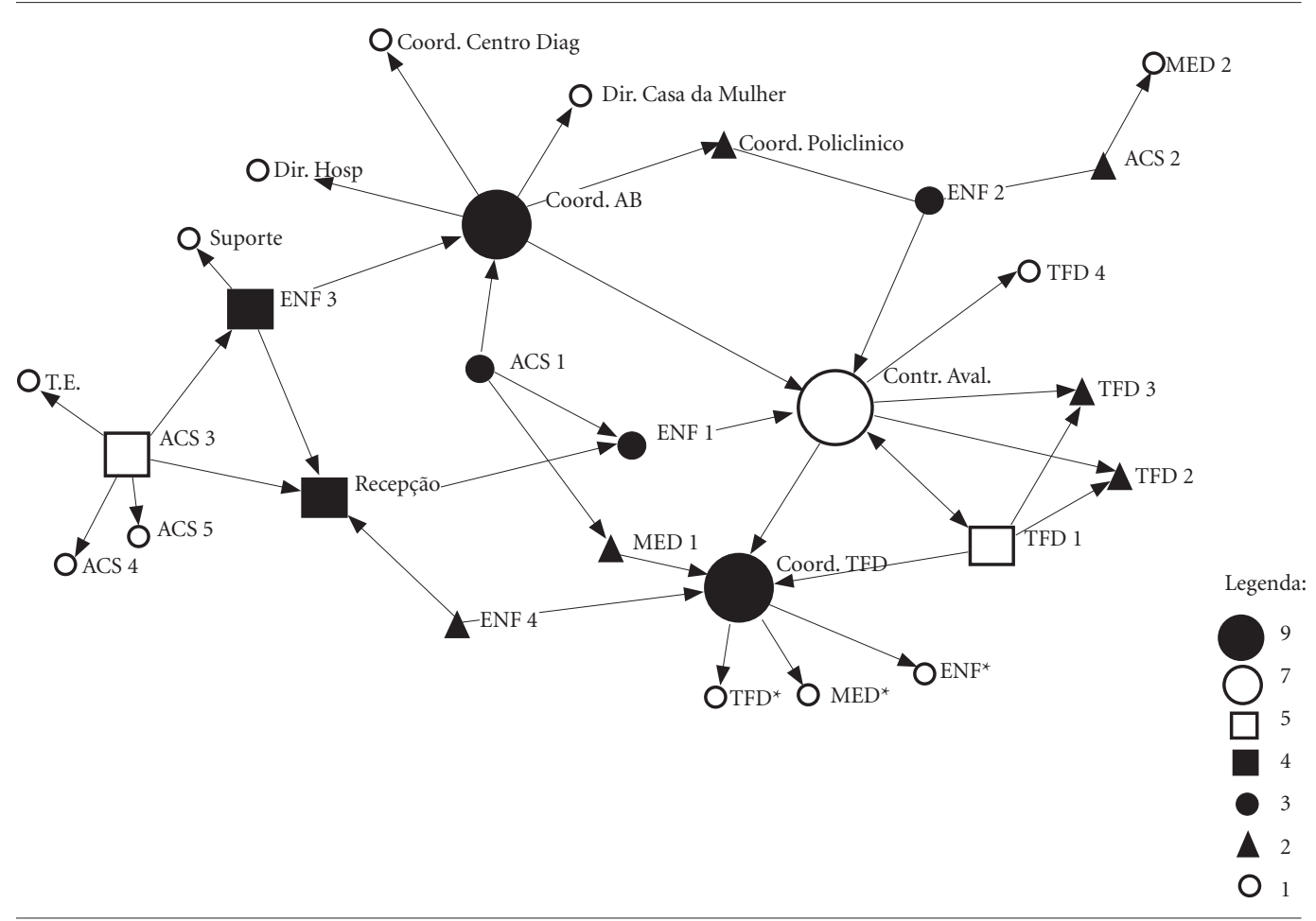

Figura 2. Rede social dos profissionais segundo a centralidade de grau. 
Quadro 1. Medidas de centralidade de grau dos atores envolvidos no acesso aos serviços de saúde.

\begin{tabular}{|l|c|c|c|l|c|c|c|}
\hline \multicolumn{1}{|c|}{ Atores } & $\begin{array}{c}\text { Grau de } \\
\text { entrada }\end{array}$ & $\begin{array}{c}\text { Grau de } \\
\text { saída }\end{array}$ & Grau & \multicolumn{1}{|c|}{ Atores } & $\begin{array}{c}\text { Grau de } \\
\text { entrada }\end{array}$ & $\begin{array}{c}\text { Grau de } \\
\text { saída }\end{array}$ & Grau \\
\hline Cont. Aval. & 4 & 5 & 9 & ACS2 & 0 & 2 & 2 \\
\hline Coord. AB & 2 & 5 & 7 & ENF4 & 0 & 2 & 2 \\
\hline Coord. TFD & 4 & 3 & 7 & Coord. Centro Diag. & 1 & 0 & 1 \\
\hline TFD1 & 1 & 4 & 5 & Dir. Hosp. & 1 & 0 & 1 \\
\hline ACS3 & 0 & 5 & 5 & Dir. Casa da Mulher & 1 & 0 & 1 \\
\hline Recepção & 4 & 0 & 4 & TFD4 & 1 & 0 & 1 \\
\hline ENF3 & 1 & 3 & 4 & MED2 & 1 & 0 & 1 \\
\hline ENF1 & 1 & 2 & 3 & Suporte & 1 & 0 & 1 \\
\hline ACS1 & 0 & 3 & 3 & ACS4 & 1 & 0 & 1 \\
\hline ENF2 & 1 & 2 & 3 & ACS5 & 1 & 0 & 1 \\
\hline MED1 & 1 & 1 & 2 & TE & 1 & 0 & 1 \\
\hline Coord. Policlínico & 2 & 0 & 2 & MED & & 1 \\
\hline TFD2 & 2 & 0 & 2 & ENF & 1 & 0 & 1 \\
\hline TFD3 & 2 & 0 & 2 & TFD & 1 & 0 & 1 \\
\hline
\end{tabular}

Legenda: $\left(^{*}\right)$ Profissionais de outros municípios.

Coord. AB- Coordenação da Atenção Básica; TFD- Tratamento fora de domicílio; ENF- Enfermeiro; ACS- Agente Comunitário de Saúde; MED- Médico; Contr. Aval- Controle e Avaliação; Suporte- Suporte para o didática digital (sistema informatizado utilizado dentro do município); TE- Técnico de enfermagem.

Fonte: Os autores, 2016.

problemas de saúde da população. No entanto, cada esfera de governo possui responsabilidades na garantia do acesso, e o modo de atuação principalmente dos municípios divergem de acordo com suas particularidades.

No município estudado, cujo acesso aos serviços é regulado por cotas, o controle e avaliação, entre outras atribuições, distribui e coordena cotas para cada profissional com base na população adscrita e nas necessidades de saúde de cada área e de cada munícipe. Trata-se de uma estratégia que é justificada pelo próprio setor como um recurso para garantia da qualidade da assistência e para o atendimento satisfatório das necessidades de saúde dos usuários.

Este não costuma ser um achado usual, já que é justamente o sistema de referência e contra-referência entre níveis uma das grandes dificuldades para o acesso e resolubilidade no SUS em municípios pequenos ${ }^{24}$. Deve-se considerar, no entanto, que os arranjos organizativos do nível local podem conseguir, em alguns casos, estabelecer relações orgânicas e sistemáticas entre os setores e níveis, atendendo de modo satisfatório a estas necessidades, o que parece ser o caso deste município.

O município conseguiu, a partir do aumento da sua situação econômica, estruturar uma rede de referência entre a atenção básica e outros níveis relativamente eficazes para a maioria dos problemas encaminhados, ficando poucos casos para serem referenciados a outros municípios, não havendo também menção a conflitos entre gestores na Região Médio Paraíba que pudessem afetar $\mathrm{o}$ atendimento aos munícipes.

Como o controle e avaliação possui grau de centralidade 9 , significa que possui maior número de contatos diretos e, assim, possui prestígio e influência na rede, é um ator "popular e expansivo"18. Caso a regulação dentro do município não consiga atender as demandas,o controle e avaliação repassará ao TFD. Desta forma, é possível garantir o acesso em outras unidades de saúde fora do município, para que haja a continuidade do cuidado. Isso porque, conforme o decreto $\mathrm{n}^{\circ} 8.901 / 2016$ compete ao departamento de regulação, controle e avaliação gerir os sistemas de informação do SUS no que se refere às macrofunções de [...] regulação da atenção e do acesso à assistência $[. . .]^{25}$.

Quanto ao grau de entrada, percebe-se que três enfermeiros e o TFD1 citaram o Controle e avaliação. Este dado permite concluir que os enfermeiros lançam mão desta possibilidade para garantir e agilizar o acesso aos serviços especializados dos usuários. Tais enfermeiros possuem potencial de obter vantagens na rede, em relação ao acesso, por não precisarem de intermediadores e por estarem em contato direto com um ator central.

Resultados de um estudo de redes sociais, publicado no Canadá em 2013, sobre a atuação 
de enfermeiros na Atenção Primária logrou resultados similares, com métricas de centralidade de grau e intermediação altas para esta categoria profissional $^{21}$. As conclusões do estudo, que analisou a estrutura dos comportamentos de troca de conhecimentos, conferiu ao enfermeiro um papel de "mediador entre fronteiras do conhecimento" 21 .

O TFD, por sua vez, é um setor estratégico de garantia ao acesso à atenção especializada para que os usuários tivessem a garantia de tratamento e cuidados em saúde quando, em seus municípios, os recursos se esgotarem. O MS lançou a portaria no 55 , de fevereiro de 1999, que dispõe sobre o trabalho do TFD garantindo, além da assistência em outro município, os recursos necessários para o usuário, como estadias, alimentação e transporte, ou seja, auxiliando-os nas despesas ${ }^{26}$.

Em função da estrutura adequada de serviços e a busca do cumprimento das normativas pela gestão municipal, justifica-se a relevância deste setor e seu aparecimento em destaque na Figura 2, com grau de entrada 4. Muitos entrevistados citaram a coordenação do TFD como um ator importante para garantir o acesso aos níveis de média e alta complexidade. Este resultado precisa ser problematizado, no entanto, em função de os dados do estudo não serem suficientes para uma avaliação mais extensiva quanto à efetiva resolutividade das ações de referência entre os níveis da Atenção Básica e de média e alta complexidades, para além das opiniões dos entrevistados. Este é, também, um limite metodológico dos estudos de redes sociais de base estrutural: se, por um lado, permitem mapear atores sociais e suas posições nas redes, por outro, são sempre um retrato pontual da realidade, que não capta a dinâmica de reconfiguração e trocas constante das redes sociais ${ }^{10}$.

Outro nó com maior centralidade de grau é o enfermeiro coordenador da atenção básica que ocupa a função há aproximadamente dois anos (data da coleta de dados). Apesar do pouco tempo nesta coordenação, este profissional possui longa trajetória de trabalho no SUS do município, tendo ocupado diversos cargos, além de possuir um perfil comunicativo e assertivo ${ }^{19}$.

É notório que, dentre as categorias profissionais que atuam no SUS, é a enfermagem a que mais tende a ocupar os espaços de gerência e gestão local ${ }^{19}$. Isto se explica pelo caminhar histórico e pela constituição da prática da enfermagem em duas dimensões indissociáveis, a do cuidado ao usuário e a do cuidado à instituição. Esta duplicidade sustenta a divisão técnica do trabalho entre enfermeiros e técnicos/auxiliares de enfer- magem, e se desdobra em diferentes modelos de organização do trabalho. Estes expressam as características do contexto em que se desenvolvem e os arranjos organizacionais possíveis ${ }^{27}$.

Há, de forma geral, o predomínio de um modelo funcional de gestão do cuidado e do trabalho pelo enfermeiro, com foco na resolução de problemas, com fragmentação e parcelização das tarefas, em contraponto à ideia de um modelo integral de cuidado, no qual o planejamento e a execução das ações se dariam de forma integrada, com etapas inter-relacionadas, e minimizando a divisão hierárquica do trabalho em equipe. O que se tem observado, no entanto, é que o enfermeiro que atua na Atenção Básica assume uma multiplicidade de tarefas e possui importante papel mediador entre o usuário e a capacidade de resposta dos serviços, e que acaba por ser também um facilitador do trabalho dos demais profissionais ${ }^{27}$.

Ao observar a posição ocupada na rede pelo enfermeiro coordenador da atenção básica (Figura 2), percebe-se que há sete ligações diretas com ele, o que o faz ter um destaque maior em se tratando de centralidade de grau. Dentre esses links, dois representam o grau de entrada, sendo um enfermeiro e um ACS, os quais exercem papéis importantes na liderança para a atenção básica, como afirma Silva ${ }^{28}$ :

São dois personagens que assumem papel de líder da equipe no Programa Saúde da Família (PSF), sendo eles o enfermeiro e o agente comunitário da saúde, pois, respectivamente, um por ocupar o cargo gerencial e administrativo e o outro exerce liderança a partir do conhecimento que possui da realidade da equipe e das comunidades ${ }^{28}$.

Essa posição na rede é vantajosa para ambas as partes; para a coordenação, que recebe informação direta de outros enfermeiros das unidades e da comunidade, por intermédio do contato com o ACS. E também, o enfermeiro pode obter privilégios para a unidade ou para o usuário ao manter contato com um elo central, que está conectado a diretores e coordenadores de outros níveis de atenção à saúde dentro do município.

A alta expansividade e influência na rede do coordenador da atenção básica resulta dos contatos diretos com coordenadores e gestores das unidades de serviço de saúde, da atenção primária à terciária ao longo dos anos. Além disso, conhece e utiliza espaços de negociações regionais e reuniões de grupo técnico para formar outros laços, ampliando sua rede de interações. Assim, na inexistência de recursos suficientes dentro do município, percebe-se, a partir do estudo, que desde a sua rede social este profissional é capaz 
de identificar e mobilizar novos recursos para garantia do acesso dos usuários ${ }^{19}$.

Ao incorporar tantas atividades e ao assumir um papel central de gestor e mediador na sua prática, o enfermeiro busca a construção de uma identidade, na qual o seu núcleo de saber encontre, principalmente, o reconhecimento profissional por parte dos indivíduos, dos grupos sociais, das demais categorias e da gestão.

A atuação do enfermeiro na rede, se dá, como em qualquer rede social, num jogo de relações, no qual estão em confronto saberes e capitais diversos ${ }^{10}$. Assim, os capitais informacionais acumulados ao longo dos muitos anos de atuação do enfermeiro coordenador da atenção básica parecem ter lhe conferido um certo prestígio e capacidade de controle dos fluxos de informação para a tomada de decisões, o que é facilitado também pela existência de uma rede local e regional que responde às demandas.

\section{Centralidade de intermediação}

A centralidade de intermediação é uma métrica que permite a mensuração da importância de um ator na rede a partir do quanto ele intermedeia as relações, ou seja, avalia o quanto um vértice está no caminho geodésico entre dois outros vértices, isto é, analisa a importância do vértice na passagem de informação entre outros dois $^{29,30}$. Caminho geodésico significa o menor caminho entre dois nós quaisquer da rede.

Um ator com alta centralidade de intermediação possui grande potencial de controle do fluxo de informação na rede. Por isso, pode influenciar na comunicação dessa rede e, ainda, como um ator-ponte, tem o potencial de conectar grupos diferentes e, caso este tenha alta centralidade de intermediação, pode desconectar a rede caso seja excluído dela ${ }^{22,31}$.

Nesta rede (Figura 3), o tamanho dos símbolos para os nós indica a centralidade de intermediação - quanto maior, maior a centralidade de intermediação. Os atores com alto índice de centralidade de intermediação são: controle e avaliação e coordenador da atenção básica, seguidos pelos atores: coordenador do TFD, enfermeiros 2 e 3, recepção e ACS 3. São os atores centrais que controlam e exercem influência na comunicação entre dois nós quaisquer que o utilizam como intermediário. Ou seja, são importantes mediadores que possuem certo poder na rede, como controlar o fluxo de informação, recursos e tudo o que precisar ser passado para outro ator o utilizando como 'ponte', como um elo de ligação ${ }^{32}$.
Assim como na centralidade de grau, os atores com maior destaque são gestores responsáveis pelo acesso aos serviços de saúde. Isto ocorre pelo fato do estudo ser sobre rede social para a regulação do acesso, porém outros atores também aparecem como centrais em relação à intermediação, principalmente enfermeiros e ACS.

Entende-se que os enfermeiros, são atoreschave e articuladores comprometidos com a integralidade das ações e com as necessidades de saúde dos usuários. Como articuladores, são capazes de atuar como instrumento de mudança, em busca da consolidação de novas práticas de saúde ${ }^{27}$. Para isso, faz-se necessário que esses profissionais façam a gestão do cuidado, aproximando as ações gerenciais das assistenciais. Embora seja preconizado e normatizado que o enfermeiro "promova a interação com outros profissionais e ocupe espaços de articulação e negociação a favor da garantia da qualidade do cuidado" 27 , os arranjos locais e os diversos capitais em jogo é que definirão as possibilidades de efetivar um cuidado integrador.

Outro profissional conhecido por seu trabalho como articulador e mediador é o ACS. Seu ofício é pautado nas relações e na interação. Atuam mediando as comunicações entre as equipes de saúde e a comunidade na qual estão inseridos, medeiam relacionamentos e facilitam a comunicação dos usuários com os serviços. O ACS é um profissional de saúde inserido na comunidade que conhece as realidades e cotidianos dos usuários facilitado pelo seu pertencer a esta realidade. Essa inter-relação entre ACS e usuário confere à unidade de saúde a vantagem de diálogo o mais próximo possível da população ${ }^{33}$.

O fato de os ACS não terem surgido na análise de centralidade de grau, mas terem importância quanto à centralidade de intermediação chama a atenção para dois aspectos, já bastante discutidos, a respeito deste ator profissional: baixo reconhecimento profissional, dentro das equipes, e potencialidade para atuar como mediador importante junto aos usuários, nos territórios ${ }^{34}$.

Outro aspecto que tem sido observado em estudos recentes, de redes sociais na atenção básica, quanto ao papel do ACS, indica que este tende a ocupar uma posição não central, expressa pelo termo "elo fraco" para classificar os atores que, nas métricas de centralidade, aparecem na periferia dos sociogramas ${ }^{13,15}$. Em análise de redes sociais, o termo "fraco" não deve confundir ou impor uma única interpretação. O elo fraco é justamente aquele que garante a expansividade da rede, pela sua capacidade de estabelecer relações com outras 


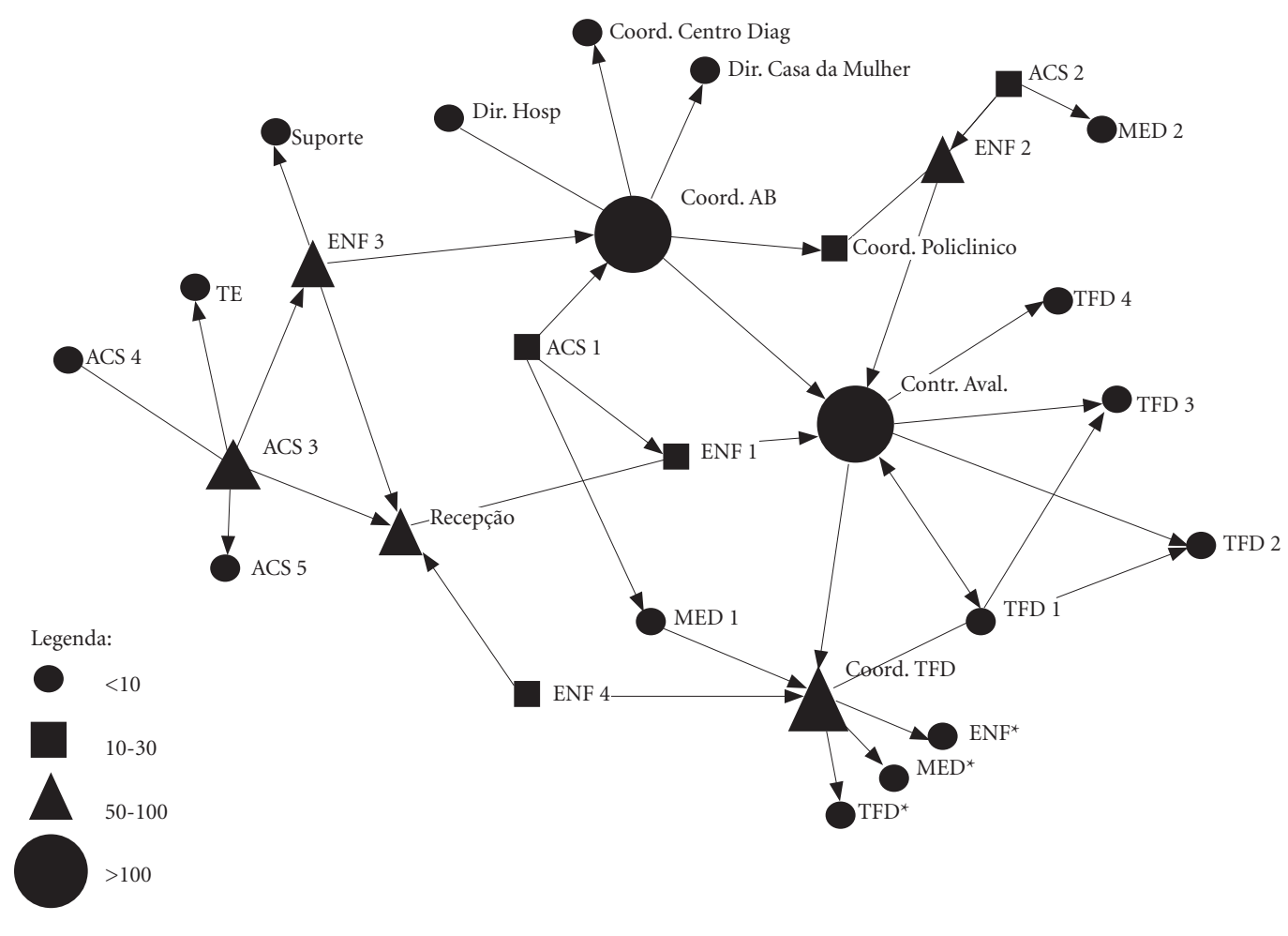

Figura 3. Rede social de atores conforme a medida de centralidade de intermediação.

Fonte: Autores, 2016.

redes, o que também é apontado como um aspecto que fortalece uma rede, dado que se esta não se expande ou renova, tende a se desintegrar ${ }^{13}$.

Atores com maior centralidade tendem a desconectar a rede caso sejam excluídos dela. Isto acontece quando se analisa apenas a representação gráfica, pois como as redes são dinâmicas, elas irão se reconfigurar assumindo nova conformação e outras ligações surgirão. Essa relevância estrutural, que conecta diferentes grupos e atores que outrora poderiam estar isolados pode ser percebida na Figura 4, em que se utilizou a medida cutpoint, que calcula as posições dos atores quanto à capacidade de desconectar a rede caso seja dela removido. Os atores com potencial de desconectar a rede estão destacados na figura em quadrados pretos.

Estes são os atores intermediadores, são eles quem conectam a rede internamente, e também estabelecem elos com outras redes. Por meio dessa conexão, informações circulam e não ficam limitadas a um grupo ou um único ator. Com a rede desconectada, há limitação na troca de diversos recursos e, considerando os serviços de saúde, estes não conseguiriam atender à demanda da população nem poderiam prestar uma assistência de qualidade. Pode-se considerar que, com uma suposta desconexão, o acesso aos serviços seria prejudicado, uma vez que não haveria comunicação, contatos e elos para servir de intermediadores, ainda que temporariamente.

Pode-se perceber que os nós destacados em preto, ao serem desligados da rede acabam por desconectar outros atores, tendendo a formar pequenos grupos também isolados, os clusters $^{10}$. Unidades de saúde dentro do município que atuam na atenção à saúde em níveis secundário e terciário ficariam desconectadas da rede ao excluir a coordenação de Atenção Básica que faz esse elo. Lembramos que é este profissional que, diante de alguma demanda, contata a atenção especializada no município para garantir o acesso a algum usuário que necessite.

Ressalta-se ainda, a conexão de três equipes da atenção básica apontando a relevância do setor de recepção. Embora esta não esteja destacada 


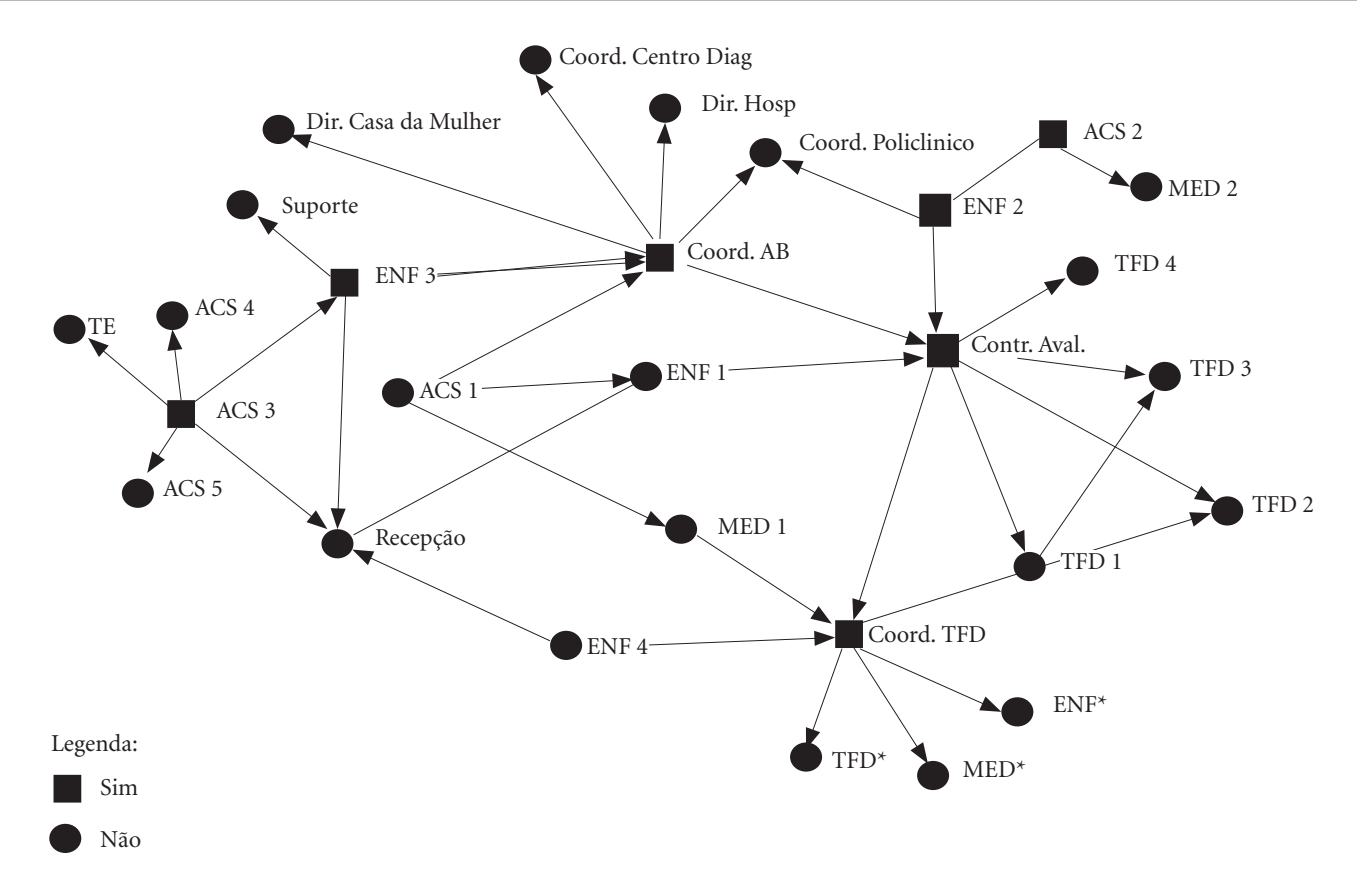

Figura 4. Atores com potencial para desconectar a rede (cut point).

Fonte: Autores, 2016.

na imagem do sociograma, é um elo importante que garante maior rapidez na comunicação entre as equipes. No município, os profissionais deste setor demonstram estar capacitados e preparados para receber diferentes demandas e usuários de diferentes faixas etárias.

Além disso, sinais evidentes de maior risco ou sofrimento, expressos pelos usuários, têm sido identificados pelos trabalhadores da recepção, que acionam profissionais com competência técnica e capacidade resolutiva de acordo com cada demanda,contribuindo para a facilitação do acesso, e gerando as vocalizações de satisfação pelos profissionais. Os resultados levam a crer que os usuários também iriam expressar algum grau de satisfação com as respostas caso fossem entrevistados, o que, no entanto, não é possível se avaliar.

\section{Conclusão}

O estudo analisou as redes sociais nos espaços de trabalho da Atenção Básica no qual possibilitou identificar o papel dos atores para garantia do acesso às redes de atenção especializada, em especial quando há insuficiência de recursos formalmente estruturados.
A situação suis generis do município estudado acabou por se constituir em uma surpresa, pelo seu perfil socioeconômico e pela existência de uma adequada rede de serviços. Esta não tem sido a realidade mais frequentemente encontrada nos muitos estudos realizados na Atenção Básica. Ainda assim, esta especificidade nos permitiu efetivar uma análise na qual foi possível destacar atributos da rede em si, já que muitas interferências e problemas causados pela baixa eficácia dos serviços pareciam, no momento do estudo, estar satisfatoriamente contempladas pela gestão.

No entanto, já assinalamos os limites desta metodologia, em especial o fato de as redes mapeadas se constituírem numa representação estática das relações sociais, exigindo um aprofundamento sobre o perfil dos atores e as formas relacionais.

Os atributos da rede de profissionais analisados no estudo permitiram identificar com maior clareza aspectos relativos às características e ao processo de trabalho de duas categorias de trabalhadores, enfermeiros e ACS. Os profissionais médicos são mencionados na rede, embora sem atributos de centralidade relevantes. Chama a atenção a não menção a quaisquer outras categorias profissionais. 
É importante ressaltar que as recentes mudanças na PNAB, publicadas na Portaria $n^{\circ}$ $2.436 / 17$, de 2017, poderão, num futuro próximo, ocasionar impactos nas redes de serviço. A portaria que traz mudanças com relação ao prazo de implantação das equipes, à cobertura do Núcleo Ampliado de Saúde da Família e Atenção Básica (NASF-AB), e prevê a alteração da equipe mínima da Estratégia Saúde da Família (ESF), estabelecendo a possibilidade de os municípios substituírem os agentes comunitários de saúde (ACS) por auxiliares ou técnicos de enfermagem $^{35}$, marca um retrocesso em relação as conquistas da atenção básica, vindo a influenciar nas relações desses atores com a população no que diz respeito a formação de vínculo.

A mudança no modelo de atenção da equipe mínima da ESF, com a substituição dos ACS, nos direciona para um modelo biomédico e medicalizado, ligando o aumento da capacidade resolutiva da atenção básica a procedimentos técnicos ${ }^{36}$.

A portaria ainda é pauta de mobilizações populares e propostas a serem implementadas, fomentando grandes discussões que poderão ter seus impactos mensurados nas relações a partir de seu cumprimento. Nesse sentido, o retorno ao mesmo município e às mesmas unidades de saúde para um novo mapeamento da rede, para o acesso do usuário aos serviços especializados, surge como um horizonte de interesses para o andamento das pesquisas nessa área.

\section{Colaboradores}

Participaram da concepção, design, interpretação e análise dos resultados, os autores JSA Fonseca e HMSL David. Contribuíram com a redação do artigo, metodologia e revisão crítica: JSA Fonseca, TF Silva e TCS Ramos. Contribuíram realizando revisão do texto final e ajustes das referências, os autores JSA Fonseca, HMSL David, ACL Neves e RB Miranda.

\section{Referências}

1. Acioli S. Redes sociais e teoria social: revendo os fundamentos dos conceitos. Informação \& Informação 2007; 12(n. esp.):8-19.

2. Stotz EN. Redes sociais e saúde. In: Marteleto RM, Stotz $\mathrm{EN}$, organizadores. Informação, saúde e redes sociais: diálogos de conhecimentos nas comunidades da Maré. Rio de Janeiro: Fiocruz; 2009. p. 27-42.

3. Recuero RC. Redes sociais online $x$ Rede sociais off-line [Internet]. Blog Social Media. Agosto de 2009 - [acessado 2016 Nov 18]. Disponível em: http://www.raquelrecuero.com/arquivos/redes_sociais_online_x_redes_sociais_offline.html

4. Marteleto RM. Redes sociais, mediação e apropriação de informações: situando campos, objetos e conceitos na pesquisa em Ciência da Informação. Ci. Inf. [periódico na Internet]. 2010 [acessado 2016 Jul 07]; 3(1):2746. Disponível em: http://inseer.ibict.br/ancib/index. php/tpbci/article/view/26/56

5. Mangia EF, Muramoto MT. O estudo de redes sociais: apontamentos teóricos e contribuições para o campo da saúde. Rev. Ter. Ocup. [periódico na Internet]. 2005 [acessado 2016 Jul 07]; 16(1):22-30. Disponível em: http://www.revistas.usp.br/rto/article/view/13955
6. Laurell AC. La salud-enfermedad como proceso social. Cuad. méd. soc. [periódico na Internet]. 1982 Jan [acessado 2016 Jul 09];19:7-20. Disponível em: http:// www.ccgsm.gob.ar/areas/salud/dircap/mat/matbiblio/ laurell.pdf

7. Albuquerque MSV, Lima LP, Costa AM, Melo Filho DA Regulação assistencial no recife: possibilidades e limites na promoção do acesso. Saude Soc 2013;22(1):223-236.

8. Brasil. Portaria ${ }^{\circ} 1.559$, de 1 de agosto de 2008. Institui a Política Nacional de Regulação do Sistema Único de Saúde - SUS. Diário Oficial da União 2008; 01 ago.

9. Silva BFS, Benito GAV. A voz de gestores municipais sobre o acesso à saúde nas práticas de gestão. Cien Saude Colet 2013; 18(8):2189-2200.

10. Marteleto RM, Tomaél MI. A metodologia de análise de redes sociais. In: Valentim MLP, organizadora. Métodos qualitativos de pesquisa em Ciência da Informação. São Paulo: Polis; 2005. p. 81-100.

11. Instituto Brasileiro de Geografia e Estatística (IBGE). Indicadores sociais municipais: uma análise dos resultados do universo do censo demográfico. Rio de Janeiro: IBGE; 2011. 
12. Brasil. Ministério da Saúde (MS). Monitoramento de indicadores de DCNT. Brasília: MS; 2016.

13. Borgatti SP, Everett MG, Freeman LC. Ucinet for Windows: Software for Social Network Analysis [computer program]. Version 6.647. Harvard: Analytic Technologies; 2002.

14. Brandes U, Borgatti SP, Freeman LC. Maintaining the Duality of closeness and betweenness centrality. Social Networks [serial on the Internet]. 2015 [cited 2017 Jun 23];44:153-159. Available from: http://www.sciencedirect.com/science/article/pii/S0378873315000738

15. Borgatti SP. NetDraw Software for Network Visualization [computer program]. Version 6.647. Lexington: Analytic Technologies; 2002.

16. Bez GS, Faraco RA, Angeloni MT. Aplicação da técnica de Análise de Redes Sociais em uma Instituição de Ensino Superior. In: XXVI simpósio de gestão da inovação tecnológica;2010; Vitória. p. 1-16.

17. Alejandro VAO, Norman AG. Manual introductorio al análisis de redes sociales. Web Redes [serial on the Internet]. 2005 Jun [cited 2017 Jun 13]:1-45. Available from: http://revista-redes.rediris.es/webredes/talleres/ Manual_ARS.pdf

18. Vesco DGD, Scarpin JE, Machado DG. Cooperação entre os Capitais Sociais em Empresas Concessionárias de Serviços Públicos no Brasil: Evidências Estruturais em Concessões de Rodovias. In: XXXIV Encontro da ANPAD; 2010; Rio de Janeiro. p. 1-15.

19. Fonseca JSA. Redes sociais na regulação da assistência à saúde em um município de pequeno porte do Rio de Janeiro [dissertação]. Rio de Janeiro: Universidade do Estado do Rio de Janeiro; 2017.

20. Hausmann M, Peduzzi M. Articulação entre as dimensões gerencial e assistencial do processo de trabalho do enfermeiro. Texto Contexto Enferm 2009; 18(2):258265.

21. Quinlan E, Robertson S. The communicative power of nurse practitioners in multidisciplinary primary healthcare teams. J Am Assoc Nurse Pract 2013; 25(2):91-102.

22. Tomaél MI, Marteleto RM. Redes sociais: posições dos atores no fluxo da informação. Encontros Bibli: revista eletrônica de biblioteconomia e ciência da informação [periódico na Internet]. 2006 [acessado 2016 Nov 02]; (n. esp). Disponível em: https://periodicos.ufsc.br/index.php/eb/article/view/15182924.2006v1 lnesp1p75

23. Brasil. Portaria no 423 , de 24 de junho de 2002 . Dispõe sobre o detalhamento das atribuições básicas inerentes a cada nível do Governo no controle, regulação e avaliação da Assistência à Saúde no SUS. Diário oficial da União 2002; 24 jun.

24. Medeiros CRG, Gerhardt TE. Avaliação da Rede de Atenção à Saúde de pequenos municípios na ótica das equipes gestoras. Saúde em Debate [periódico na Internet]. 2015 [acessado 2018 Jan 28]; 39(n. esp.):160-170. Disponível em: https://doi.org/10.5935/ 0103-1104.2015S005201.

25. Brasil. Decreto no 8.901, de 10 de novembro de 2016. Aprova a Estrutura Regimental e o Quadro Demonstrativo dos Cargos em Comissão e das Funções de Confiança do Ministério da Saúde, remaneja cargos em comissão e funções gratificadas e substitui cargos em comissão do Grupo Direção e Assessoramento Superiores - DAS por Funções Comissionadas do Poder Executivo - FCPE. Diário oficial da União 2016; 10 nov.
26. Brasil. Portaria no 55 , de 24 de fevereiro de 1999. Dispõe sobre a rotina do Tratamento Fora de Domicilio do Sistema Único de Saúde - SUS, com inclusão dos procedimentos específicos na tabela de procedimentos do Sistema de Informações Ambulatoriais do SIA/SUS e dá outras providências. Diário oficial da União 1999; $24 \mathrm{fev}$.

27. Peduzzi M, Silva AM, Lima MADS. Enfermagem como prática social e trabalho em equipe. In: Soares CB, Campos CMS, organizadores. Fundamentos de saúde coletiva e o cuidado de enfermagem. Barueri: Manole; 2012. p. 217-243.

28. Silva FHC. A atuação dos enfermeiros como gestores em Unidades Básicas de Saúde. Revista de Gestão em Sistemas de Saúde 2012; 1(1):67-82.

29. Faria LHL, Giuliane AC, Cunha CF, Souza MAVF, Freitas RS. A produção científica em comportamento do consumidor no Brasil de 2007 a 2011, sob a perspectiva da análise das redes sociais (ARS). Revista Educação, Tecnologia e Cultura [periódico na Internet]. 2016 [acessado 2016 Nov 12]; 13(1):1-18. Disponível em: http://www.publicacoes.ifba.edu.br/index.php/etc/article/view/5/14

30. Del-vecchio RR, Galvão DJC, Silva L, Lima RFVL. Medidas de Centralidade de Teoria dos Grafos aplicada a Fundos de Ações no Brasil. In: XLI SBPO - Simpósio Brasileiro de Pesquisa Operacional; 2009; Niterói. p. 533-545.

31. Marteleto RM. Análise de redes sociais - aplicação nos estudos de transferência da informação. Ci. Inf. 2001; 30(1):71-81.

32. Izquierdo LR, Hanneman RA. Introduction to the formal analysis of social networks using mathematica. Riverside [serial on the Internet]. 2006 [cited 2017 Jan 30] p.1-29. Available from: http://citeseerx.ist.psu.edu/ viewdoc/download?doi=10.1.1.722.9695\&rep=rep1\&type $=$ pdf

33. Pinheiro RL, Guanaes-lorenzi C. Funções do agente comunitário de saúde no trabalho com redes sociais. Estudos de Psicologia 2014; 19(1):48-57.

34. Silva TF, Romano VF. Sobre o acolhimento: discurso e prática em Unidades Básicas de Saúde do município do Rio de Janeiro. Saúde em Debate 2015; 39(5):363-374.

35. Brasil. Portaria no 2.436, de 21 de setembro de 2017. Aprova a Política Nacional de Atenção Básica, estabelecendo a revisão de diretrizes para a organização da Atenção Básica, no âmbito do Sistema Único de Saúde (SUS). Diário oficial da União 2017; 22 set.

36. Morosini MVGC, Fonseca AF. Revisão da Política Nacional de Atenção Básica numa hora dessas? Cad Saude Publica 2017; 33(1):1-3.

Artigo apresentado em 29/01/2018

Aprovado em 06/03/2018

Versão final apresentada em 05/06/2018 